Check for updates

Cite this: RSC Adv., 2017, 7, 19754

\title{
Fabrication of a silica/titania hollow nanorod and its electroresponsive activity $\dagger$
}

\begin{abstract}
Chang-Min Yoon, Jungchul Noh, Yoonsun Jang and Jyongsik Jang (DD *
In this study, a 1D oriented hollow $\mathrm{SiO}_{2} / \mathrm{TiO}_{2}$ (HST) rod-like material was successfully fabricated via a sequential combination of sol-gel use, $\mathrm{TiO}_{2}$ incorporation, and a sonication-mediated etching and redeposition method. This carefully manipulated new material has numerous advantageous physical and intrinsic properties, such as increased surface area, pore volume, interfacial polarization, and dielectric properties introduced from each synthetic step. The synthesized HST rod was adopted as an electrorheological (ER) material for practical examination of these characteristics. The HST rod materials exhibited 1.5- and 3-fold higher ER performance than a non-metal $\mathrm{SiO}_{2}$ rod and a non-hollow $\mathrm{SiO}_{2} / \mathrm{TiO}_{2}$ core/shell (ST/CS) rod, which are interim synthetic steps. Moreover, the HST rod exhibited remarkable 6 -fold increased ER efficiency relative to a sphere-shaped hollow $\mathrm{SiO}_{2} / \mathrm{TiO}_{2}$ particle synthesized using a similar experimental method. These notable enhancements in ER performance are attributed to incorporation of the experimentally designed characteristics of the HST rod: 1D structure, metal oxide incorporation, and creation of a hollow cavity. For future study, we expect that these versatile HST rod materials can be applied in a range of fields including drug delivery, photo-catalysis, and as building blocks.
\end{abstract}

Received 13th February 2017 Accepted 27th March 2017

DOI: $10.1039 / \mathrm{c} 7 \mathrm{ra01786c}$

rsc.li/rsc-advances such as carbon fibers, metals, metal oxides, silicon, and their hybrids. ${ }^{9-12}$

Among these inorganic materials, metal oxides are of great interest because they are among the most abundant minerals on Earth. ${ }^{13}$ Metal oxides are ionic compounds consisting of a positive metal component and a negative oxygen ion, resulting in a chemically and thermally stable molecule. However, most of the distinct properties of metal oxides arise from the unfilled dorbital and unpaired electrons; these result in metal oxides (e.g. $\mathrm{ZnO}, \mathrm{Cu}_{2} \mathrm{O}, \mathrm{NiO}, \mathrm{V}_{2} \mathrm{O}_{5}, \mathrm{Fe}_{2} \mathrm{O}_{3}$, and $\mathrm{TiO}_{2}$ ) having unique properties such as photoconduction, catalytic activity, a wide bandgap, and a high dielectric constant. ${ }^{\mathbf{1 4 - 1 6}}$ Nevertheless, controlling the anisotropic growth of metal oxides and manipulating a target 1D structure remain major challenges. One method for resolving difficulties in the synthetic process and taking advantage of metal oxides is to incorporate metal oxides into nonmetaltemplate materials such as a carbon substrate or $\mathrm{SiO}_{2} \cdot{ }^{17,18}$

Among metal oxides, titania $\left(\mathrm{TiO}_{2}\right)$ nanoparticles have been widely studied owing to their excellent characteristics, including high stability, corrosion resistance, dielectric properties, and photocatalytic activity. ${ }^{\mathbf{1 9 - 2 1}}$ Various methods for the fabrication of $\mathrm{TiO}_{2}$ nanoparticles have been developed, including co-precipitation, electrospray deposition, spray pyrolysis, and sol-gel methods. ${ }^{22-24}$ Moreover, $\mathrm{TiO}_{2}$ materials can be easily composited with hard templated $\mathrm{SiO}_{2}$ to attain additional advantages, such as easy dimension control, improved light scattering, and feasibility of mass production. ${ }^{25-27}$ With further modification of a $\mathrm{SiO}_{2}$ template, $\mathrm{SiO}_{2} /$ $\mathrm{TiO}_{2}$ composite materials can achieve additional physical 
characteristics such as hollow spaces and increased porosity. ${ }^{28,29}$

Synergistic effects from the methods discussed so far can be achieved through a combination of controlling the templated structure, adding metal oxides, and introducing porosity. Although, there is few studies addressing the fabrication of hollow $\mathrm{SiO}_{2}$ rod-like material or hollow $\mathrm{TiO}_{2}$ fibers, but there were drawbacks of complex experimental procedures and unevenly structured final products. Therefore, there is a necessity for wellconstructed hollow 1D rod-like nanomaterial with tunable aspect ratio $(L / D)$ by facile method is arising. Our group has previously reported the mass scale synthesis of well-defined $\mathrm{SiO}_{2}$ rod with controllable $L / D$. Also, we have reported the fabrication of uniformly ordered various hollow metal oxide-composited nanospheres by simple procedure using sonication-mediated etching and redeposition (SMER) method. ${ }^{30-32}$ Particularly, detailed experimental conditions such as types of etchants $\left(\right.$ e.g. $\mathrm{NH}_{4} \mathrm{OH}$, $\mathrm{NaOH}$, alkaline earth metal hydroxides) and sonication times are varied with size of nanospheres and intended final products (e.g. hollow $\mathrm{SiO}_{2} / \mathrm{TiO}_{2}, \mathrm{TiO}_{2}$, alkaline earth metal-doped $\left.\mathrm{SiO}_{2} / \mathrm{TiO}_{2}\right){ }^{33-35}$ However, none of previous studies including our group have reported the fabrication of hollow metal oxide-composited rod-like nanomaterial with tunable $L / D$. Accordingly, the objective of this study was to fabricate a material combining all of the advantages of a metal oxide, a 1D orientation, and high porosity by combining well-known simple (sol-gel, modified Stöber, and SMER) methods from other and our groups with some inventive modifications according to our experimental condition.

Electrorheology (ER) is the rheological study of materials dispersed in an insulating medium under the influence of an electric field..$^{36,37}$ Under an applied electric field, randomly dispersed particles form fibril-like chains to exert a mechanical change, which can be measured in terms of shear stress (Pa) and viscosity (Pa s). ${ }^{38,39}$ According to previous studies, ER performance is greatly affected by the characteristics of the dispersed material and other factors including temperature, electric field strength, and the dispersed medium. ${ }^{40-43}$ In the last few decades, most studies have focused on the fabrication of new ER materials and controlling material properties to obtain enhanced ER performance. Thus, numerous materials with different compositions, structures, sizes, and properties have been adopted as ER materials to find the maximum efficiency. ${ }^{44-46}$ Interestingly, positive ER effects require material characteristics such as a large active surface area, low particle density, high porosity, polarizability, and high electrical conductivity, ${ }^{47-50}$ and these properties have potential for utilization in other applications such as drug delivery, electrochemical studies, and photovoltaic applications..$^{34,51,52}$ Thus, ER applications are effective methods for investigating these newly designed materials.

In this study, we report a successful method for fabricating a hollow $\mathrm{SiO}_{2} / \mathrm{TiO}_{2}$ (HST) composite rod-like material for the first time. The $\mathrm{SiO}_{2}$ rod with tunable $L / D$ fabricated by modified Stöber method reported in our previous studies were used as core material. Afterwards, a porous $\mathrm{TiO}_{2}$ shell was introduced to obtain a $\mathrm{SiO}_{2} / \mathrm{TiO}_{2}$ core/shell (ST/CS) rod. A hollow structure was obtained using the sonication-mediated etching and redeposition (SMER) method reported in our previous study. The core $\mathrm{SiO}_{2}$ rod was completely dissolved in the etchant by the high sonication energy and then some was redeposited onto the $\mathrm{TiO}_{2}$ shell to create a $\mathrm{SiO}_{2} / \mathrm{TiO}_{2}$ composite. Also, the aspect ratio $(L / D)$ of HST rod was adjustable up to $c a$. 6 by changing the length of $\mathrm{SiO}_{2}$ template. The resulting HST rod exhibited various advantages, such as a hollow morphology, 1D orientation, high surface area, high pore volume, large dielectric constant, and feasibility for mass production. Furthermore, we investigated the electrorheological activity of the HST rod to elucidate the unique properties of the material. This newly designed 1D HST rod can be applied across a range of fields for future study.

\section{Experimental}

\subsection{Materials}

Tetraethyl orthosilicate (TEOS), titanium isopropoxide (TTIP, 97.0\%), cetyltrimethylammonium bromide (CTAB), and silicone oil [poly(methylphenylsiloxane), $100 \mathrm{cST}$ ] were purchased from Aldrich Chemical Co. Hydrochloric acid ( $\mathrm{HCl}, 35.0-37.0 \%)$ and ammonium hydroxide solution $\left(\mathrm{NH}_{4} \mathrm{OH}, 28.0-32.0 \%\right)$ were purchased from Samchun Chemical Co. (Korea). Absolute ethanol (ethyl alcohol, 99.5) was obtained from Fisher Chemical Co. All chemicals were used as received.

\subsection{Synthesis of $\mathrm{SiO}_{2} / \mathrm{TiO}_{2}$ core/shell (ST/CS) rod}

Core $\mathrm{SiO}_{2}$ rod template $(L / D=3)$ was synthesized according to the modified Stöber method. ${ }^{31}$ In a typical synthesis of $\mathrm{SiO}_{2}$ rod, absolute ethanol $(6 \mathrm{~mL})$, ammonia solution $(2.1 \mathrm{~mL})$, and DI water $(140 \mathrm{~mL})$ were mixed by magnetic stirring for $5 \mathrm{~min}$. Then CTAB $(0.62 \mathrm{~g})$ was dissolved into the resulting mixture and stirred for $20 \mathrm{~min}$. Subsequently TEOS $(1.2 \mathrm{~mL})$ was injected into the as-prepared solution and the reaction proceeded for $3 \mathrm{~h}$ at room temperature. $\mathrm{SiO}_{2}$ rod particles were collected by centrifugation. To remove the remaining organic species, $\mathrm{SiO}_{2}$ rods were washed by mixture of $\mathrm{HCl}(3 \mathrm{~mL})$ and ethanol $(20 \mathrm{~mL})$ for $6 \mathrm{~h}\left(70^{\circ} \mathrm{C}\right)$. Washed $\mathrm{SiO}_{2}$ rod particles were centrifuged $(6500$ $\times g$ ) with ethanol for several times and re-dispersed into the absolute ethanol $(45 \mathrm{~mL})$ by vigorous stirring. A mixture of acetonitrile $(15 \mathrm{~mL})$ and TTIP $(9 \mathrm{~mL})$ was slowly added to the colloidal suspension of $\mathrm{SiO}_{2}$ rod using a burette. The condensation reaction proceeded for $12 \mathrm{~h}$ at $4{ }^{\circ} \mathrm{C}$ and resulting white solution was centrifuged to obtain $\mathrm{SiO}_{2} / \mathrm{TiO}_{2}$ core/shell (ST/CS) rod particles. Obtained ST/CS rod was dried in oven for overnight $\left(100{ }^{\circ} \mathrm{C}\right)$. The aspect ratio (fixed diameter of $c a .65 \mathrm{~nm}$, varying length) of core $\mathrm{SiO}_{2}$ was controlled by changing the amount of DI water in above process, resulting in fabrication of $\mathrm{ST} / \mathrm{CS}$ materials with varying aspect ratio. Sphere $\mathrm{SiO}_{2}(L / D=1)$ and two $\mathrm{SiO}_{2}$ rods $(L / D=2$ and 6) were synthesized using $25 \mathrm{~mL}$, $40 \mathrm{~mL}$, and $120 \mathrm{~mL}$ of DI water, respectively.

\subsection{Fabrication of hollow $\mathrm{SiO}_{2} / \mathrm{TiO}_{2}$ (HST) rod via SMER method}

HST rod particles were fabricated via sonication-mediated etching and re-deposition (SMER) method. ${ }^{30}$ Firstly, dried ST/ 
CS rod ( $0.5 \mathrm{~g})$ was well-dispersed in DI water $(20 \mathrm{~mL})$ by vigorous stirring for $6 \mathrm{~h}$. And then, $\mathrm{NH}_{4} \mathrm{OH}(0.2 \mathrm{M}, 40 \mathrm{~mL})$ solution was added to the ST/CS dispersion and placed in the sonicator for $5 \mathrm{~h}$. Resulting white cloudy solution was centrifuged $(6500 \times g)$ with DI water and ethanol for several times to remove the etchant solution and residues. Finally, collected HST rod was dried in oven for overnight $\left(100{ }^{\circ} \mathrm{C}\right)$. HST materials with different aspect ratio were fabricated by same synthetic method except using ST/CS materials with different lengths. The yield of HST rod was ca. $0.4 \mathrm{~g}$, by following the described procedure. Under our experimental condition, the etching process can be scaled up to 20 times to obtain 8.0 grams of HST rods in one process by increasing the amount of ST/CS rod, DI water, and $\mathrm{NH}_{4} \mathrm{OH}$ solution.

\subsection{Characterization}

The morphologies of $\mathrm{SiO}_{2}$ rod, ST/CS rod, and HST rods were analyzed by transmission electron microscope (JEM-200CX, JEOL) and field-emission scanning electron microscope (JEOL6700, JEOL) equipped with an EDS spectrometer (Inca). Elemental mapping of materials were provided with STEM (Tecnai F20, FEI) equipped with image filter (Gatan, Inc.). Additional molecular information of materials were obtained by Fourier transform infrared spectroscopy (Cary-600, Agilent). The crystalline phase of materials were investigated by X-ray diffraction analysis by M18XHF-SRA (MAC Science Co.) and AXIS-His spectroscopy (KRATOS). The pore size and surface area of materials were determined by $\mathrm{BJH}$ and BET calculation associated with $\mathrm{N}_{2}$-sorption curves (ASAP-2010, Micrometrics). Dispersion stability of materials was determined by dispersing particles into silicone oil and calculating the sedimentation ratio $(R)$. Dielectric properties of particles were measured by impedance spectroscope (Solatron-1260) coupled with interface analyzer (Solartron-1296).

\subsection{Electro-response (ER activity) of HST rod-based ER fluid}

Electro-response of $\mathrm{SiO}_{2}$ rod, ST/CS rod, and HST rods were studied by preparing samples as an electrorheological (ER) fluid. To prepare ER fluids, dried materials ( $0.3 \mathrm{~g})$ were welldispersed into silicone oil $(10 \mathrm{~mL})$ by three steps. Firstly, particles were finely grinded using a mortal and pestle. And then, grinded particles were dissolved into the silicone oil by vigorous stirring for $12 \mathrm{~h}$. Finally, ER fluids were sonicated for additional $6 \mathrm{~h}$ to attain homogeneous solution. Practical ER measurements were carried out using rheometer and accessory sets (AR 2000, TA instruments). In specific, rheometer was equipped with an insulating cup $(d=30.0 \mathrm{~mm}, h=30.0 \mathrm{~mm})$, cylinder geometry $(d=28.0 \mathrm{~mm}, h=30.0 \mathrm{~mm})$, and a high voltage generator (Trek 677B). To start a measurement, asprepared ER fluids were poured into a cup and cylinder geometry was inserted into the cup. The gap distance between the cup and geometry was fixed to $1.00 \mathrm{~mm}$. Subsequently, pre-shear $\left(3.0 \mathrm{~s}^{-1}\right)$ was applied for $10 \mathrm{~min}$ to attain equilibrium state. Finally, DC voltage was applied to examine the electro-response of $\mathrm{SiO}_{2}$ rod-, ST/CS rod-, and HST rod-based ER fluids.

\section{Results and discussion}

\subsection{Fabrication of HST rod material}

The strategic method for fabrication of the HST material is shown in Fig. 1a. First, the core $\mathrm{SiO}_{2}$ template rod was synthesized using a simple sol-gel method (modified Stöber method) with CTAB as the surfactant. After the reaction remaining organic residue was completely removed by ethanolic acid wash for uniform coating of $\mathrm{TiO}_{2}$. And then, the TTIP precursor was slowly added to the core $\mathrm{SiO}_{2}$ rod to synthesize the porous $\mathrm{TiO}_{2}$ shell-coated $\mathrm{SiO}_{2}$ rod, denoted a $\mathrm{SiO}_{2} / \mathrm{TiO}_{2}$ core/shell (ST/CS) rod, via a $\mathrm{TiO}_{2}$ condensation reaction. Finally, the $\mathrm{TiO}_{2}$ embedded ST/CS rod was etched using a $\mathrm{NH}_{4} \mathrm{OH}$ etchant with sonication to synthesize a hollow HST rod material via the SMER method. The mechanism for the $\mathrm{SiO}_{2}$ core etching process and redeposition of silica species is shown in Fig. $1 \mathrm{~b}$. The $\mathrm{SiO}_{2}$ core was partially dissolved in a solution of $\mathrm{NH}_{4} \mathrm{OH}$ etchant under basic conditions. The $\mathrm{SiO}_{2}$ core was then sequentially etched further and then released into the etchant solution as a silicic ion form of $\left[\mathrm{Si}(\mathrm{OH})_{6}\right]^{2-}$ with strong intensity driven by the sonication. The dissolved silicic species reacted with hydroxide ions $\left(\mathrm{OH}^{-}\right)$from the etchant solution and was redeposited as $\mathrm{SiO}_{2}$ and $\mathrm{SiO}_{3}$ onto the $\mathrm{TiO}_{2}$ shell through the process of Ostwald ripening. In addition, some $\mathrm{TiO}_{2}$ also dissolved in the $\mathrm{NH}_{4} \mathrm{OH}$ solution and was redeposited onto the final HST rod. However, owing to the chemical stability of $\mathrm{TiO}_{2}$, the redeposition of $\mathrm{TiO}_{2}$ was generally negligible compared with the completely dissolved $\mathrm{SiO}_{2}$ core material. ${ }^{30}$

Transmission electron microscope (TEM) analysis was carried out to investigate the morphology of the materials

a)

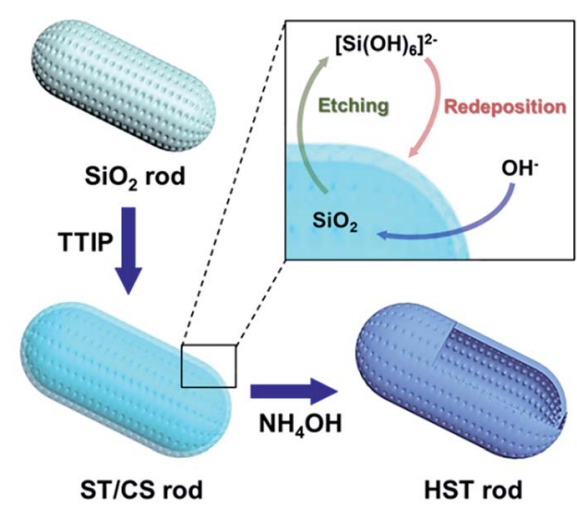

b)

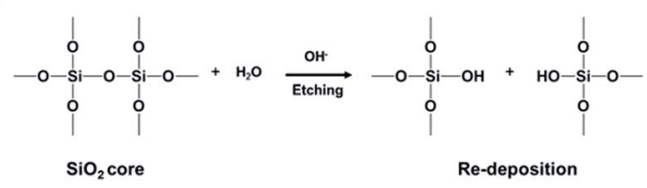

Fig. 1 (a) Schematic illustration for fabrication of HST rod via sequential steps of sol-gel, $\mathrm{TiO}_{2}$ incorporation, and sonicationmediated etching and redeposition (SMER) method. (b) Mechanism of hydroxide ion catalyst silica etching and redeposition induced in the process of SMER method. 
(Fig. 2). The diameters of the $\mathrm{SiO}_{2}, \mathrm{ST} / \mathrm{CS}$, and HST rods were determined to be $c a$. 60, $66 \pm 4$, and $72 \pm 4 \mathrm{~nm}$, and the lengths of the $\mathrm{SiO}_{2}, \mathrm{ST} / \mathrm{CS}$, and HST rods were found to be $c a$. 200, 210, and $230 \mathrm{~nm}$, respectively. Hence, the aspect ratio $(L / D)$ of three materials were determined to be $c a$. 3. The particle sizes of the ST/CS and HST rods were larger than that of the $\mathrm{SiO}_{2}$ rod owing to the formation of a porous $\mathrm{TiO}_{2}$ shell and redeposition of $\mathrm{SiO}_{2}$. The thickness of the porous $\mathrm{TiO}_{2}$ shell was determined to be $c a$. 3 to $5 \mathrm{~nm}$ and the redeposited composite shell thickness was found to be $c a$. 3 to $5 \mathrm{~nm}$. Thus, the combined shell thickness of the HST rod was $c a .8$ to $10 \mathrm{~nm}$, and a well-defined internal hollow space beneath the shell was clearly observed. SEM observations verified fairly uniform morphologies for all three materials (Fig. S1 $\dagger$ ), and an internal hollow space created within the HST rod was clearly observed from the ruptured structure (Fig. S1 $\dagger$ ). During the fabrication process, it is important to remove the remaining $\mathrm{CTAB}$ and organic residues by $\mathrm{HCl}$ treatment. Without $\mathrm{HCl}$ treatment, leftover organic residues on $\mathrm{SiO}_{2}$ rod can cause aggregation of final HST rods and formation of random $\mathrm{TiO}_{2}$ particles. Also, calcination of $\mathrm{SiO}_{2}$ rod caused aggregation of $\mathrm{SiO}_{2}$ rod and resulting HST rods. $\mathrm{HCl}$ untreated and calcined $\mathrm{SiO}_{2}$ rods and resulting HST rods are presented in Fig. S2. $\dagger$ Hence, ethanolic acid wash of $\mathrm{SiO}_{2}$ rod is essential step to attain well-constructed final HST rods. Also, the shell thicknesses of HST rods were controlled by changing the amount of added TTIP solution (Fig. S3†). However, HST rods with thin
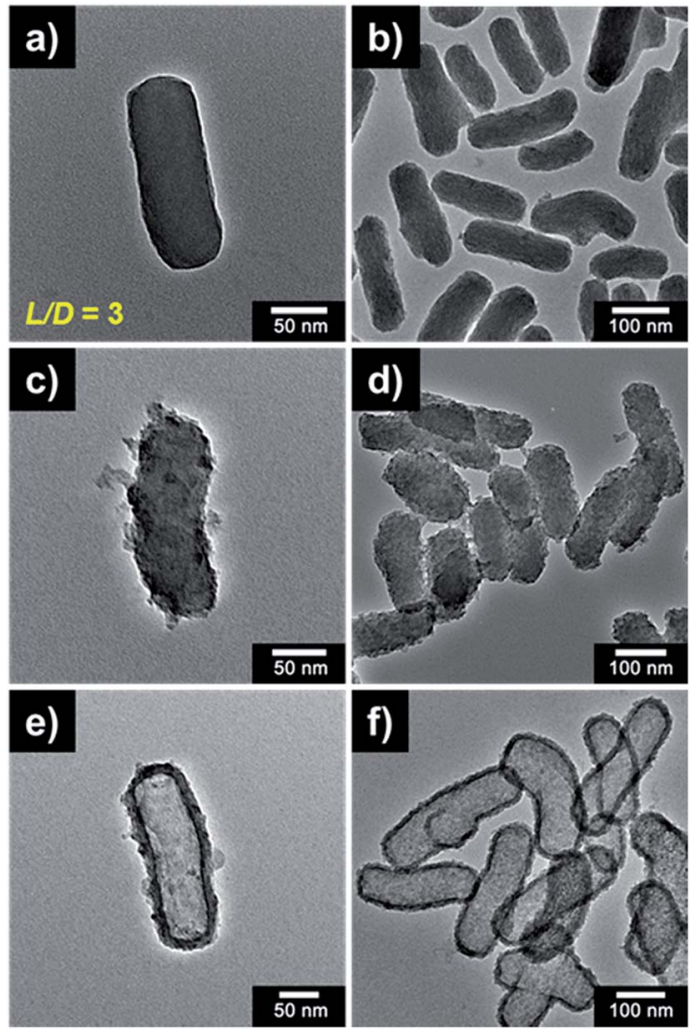

Fig. 2 TEM micrographs of (a, b) $\mathrm{SiO}_{2}$ rod, (c, d) ST/CS rod, and (e, f) HST rod, respectively $(a, b, c=$ high magnified images, $b, d, f=$ low magnified images). and thick shells had problems like aggregation, structural deformation, and blockage of etchant pathway. Moreover, the HST materials with various aspect ratios were fabricated by controlling the anisotropic growth of the core $\mathrm{SiO}_{2}$ template. Particularly, the core $\mathrm{SiO}_{2}$ materials were synthesized with fixed diameter of $c a$. $60 \mathrm{~nm}$ and only lengths were varied by changing the amount of added reagents. As a result, the HST materials from sphere-shaped $(L / D=1)$ to long $\operatorname{rod}(L / D=6)$ were prepared by same fabrication methods (Fig. S4 $\dagger$ ). However, long HST $\operatorname{rod}(L / D=6)$ showed relatively aggregated state and slight structural deformation compared to well-dispersed HST material with low aspect ratio (Fig. S5 $\dagger$ ). The particle aggregation of long HST rod is due to the increased amount of redeposited $\mathrm{SiO}_{2}$ species from elongated $\mathrm{SiO}_{2}$ template. Also, elongated structure of long HST rod could not withstand the energy driven from the sonication during the etching process resulting in some deformation of structure. Thus, the HST rod with $L / D$ of 3 is the longest rod-like material without forming particle aggregation or distorted structure under our experimental conditions. In this regard, the HST rod discussed in further context is regarded to HST rod with $(L / D=3)$. Detailed physical parameters of various HST materials are listed in Table $\mathrm{S} 1 . \dagger$

$\mathrm{N}_{2}$-sorption curves and associated Brunauer-Emmett-Teller (BET) surface area and Barrett-Joyner-Halenda (BJH) pore distributions were calculated to investigate the porosity, pore volume, and surface area of the materials, as shown in Fig. 3. The pore sizes of the $\mathrm{SiO}_{2}, \mathrm{ST} / \mathrm{CS}$, and HST rods were found to be 3.3, 4.0, and $3.8 \mathrm{~nm}$, respectively. The pore size of the HST rod was diminished compared with that of the ST/CS rod due to the redeposition of silicic species. On the other hand, the pore volume of the HST rod was significantly larger at $0.87 \mathrm{~cm}^{3} \mathrm{~g}^{-1}$ than those of the ST/CS rod $\left(0.56 \mathrm{~cm}^{3} \mathrm{~g}^{-1}\right)$ and the $\mathrm{SiO}_{2} \operatorname{rod}(0.41$

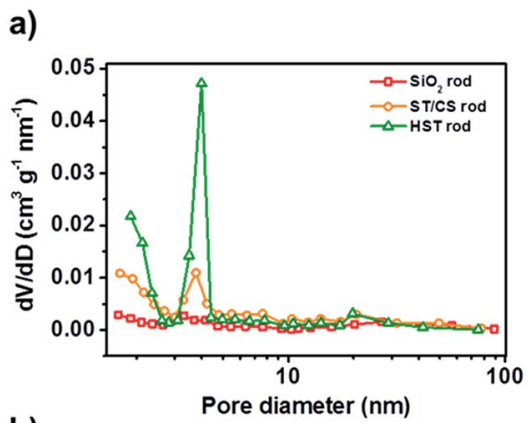

b)

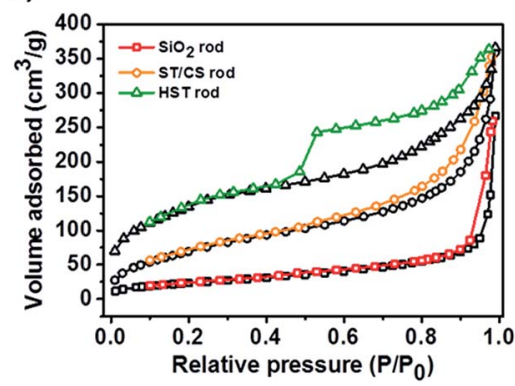

Fig. 3 (a) $\mathrm{BJH}$ pore distribution curves of $\mathrm{SiO}_{2} \mathrm{rod}, \mathrm{ST} / \mathrm{CS}$ rod, and HST rod. (b) $\mathrm{N}_{2}$-sorption curves of $\mathrm{SiO}_{2}$ rod, ST/CS rod, and HST rod. 
$\left.\mathrm{cm}^{3} \mathrm{~g}^{-1}\right)$. The increase in pore volume suggested that the core $\mathrm{SiO}_{2}$ core template was successfully etched and an internal hollow space was created within the HST rod. The $\mathrm{N}_{2}$-sorption curves of the materials confirmed the porous nature of the HST rod. While the core $\mathrm{SiO}_{2}$ rod showed a type III isotherm, the ST/ CS and HST rods exhibited typical type IV isotherms of porous materials. In particular, the hysteresis loop of the HST rod was larger than that of the ST/CS rod, indicating higher porosity. As a result, the surface area of the HST rod material was significantly larger than those of the ST/CS and core $\mathrm{SiO}_{2}$ rods owing to the creation of an internal cavity and a porous shell. Detailed physical characteristics of the rods are listed in Table S2. $\dagger$

The atomic compositions of the materials were determined by EDS analysis, as shown in Table S3. $\dagger$ In particular, the core $\mathrm{SiO}_{2}$ rods contained only $\mathrm{Si}$ and $\mathrm{O}$ elements. In contrast, Ti was detected in the ST/CS and HST rods, indicating that the $\mathrm{SiO}_{2}$ core had been successfully coated with a $\mathrm{TiO}_{2}$ shell. Notably, the Si atomic composition of the HST rod was significantly lower than that of the ST/CS rod after the core etching process; however, some $\mathrm{Si}$ atoms remained and participated in the redeposition process to obtain the final $\mathrm{SiO}_{2} / \mathrm{TiO}_{2}$ composited material. Scanning transmission electron microscope (STEM) analysis was carried out on each material (Fig. 4), and the STEM image of the $\mathrm{SiO}_{2}$ rod confirmed that the material was composed of Si elements without Ti (Fig. S6 $\dagger$ ). In the case of the ST/CS rod, Ti elements were well distributed in the outer shell and the $\mathrm{Si}$ remained inside the $\mathrm{TiO}_{2}$ shell. Interestingly, the elemental mapping image of the HST rod showed that the shell was composed of both Ti and ample amounts of Si elements distributed with the Ti elements to produce the composite state of the HST rod. The internal hollow space of the HST rod was clearly observed inside the $\mathrm{Si} / \mathrm{Ti}$ composite shell. To further investigate the molecular composition of materials, Fourier transform infrared (FT-IR) analysis were carried out (Fig. S7a $\dagger$ ). Particularly, all three samples showed characteristics peaks of $\mathrm{SiO}_{2}, \mathrm{Si}-\mathrm{O}$ stretching vibration was located near $1045 \mathrm{~cm}^{-1}$ and $\mathrm{Si}-\mathrm{O}$ bending at $940 \mathrm{~cm}^{-1}$. Also, peaks for water and organic species from synthetic process were detected at $1635 \mathrm{~cm}^{-1}$ for $\mathrm{H}-\mathrm{O}-\mathrm{H}$ bending and $3800 \mathrm{~cm}^{-1}$ for $\mathrm{O}-\mathrm{H}$ stretching. Notably, $\mathrm{TiO}_{2}$ related peaks were found from the ST/CS rod and HST rod. Two prominent $\mathrm{TiO}_{2}$ peaks were located near $1430 \mathrm{~cm}^{-1}$ and $550 \mathrm{~cm}^{-1}$, corresponding to stretch vibration of $\mathrm{Ti}-\mathrm{O}-\mathrm{Ti}$ and $\mathrm{Ti}-$

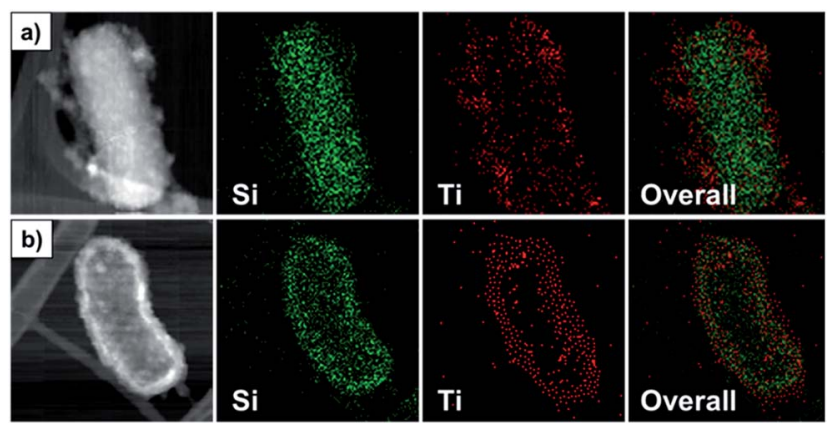

Fig. 4 STEM-HADDF image (left) and elemental mapping images of (a) ST/CS rod and (b) HST rod (detected elements: Si and Ti).
$\mathrm{O}$, respectively. ${ }^{53}$ Also, $\mathrm{Si}-\mathrm{O}-\mathrm{Ti}$ bonds were detected from the $\mathrm{ST} /$ CS and HST rod at $952 \mathrm{~cm}^{-1}$ and $947 \mathrm{~cm}^{-1}$ to indicate the successful formation of composite of $\mathrm{SiO}_{2}$ and $\mathrm{TiO}_{2}$. Additionally, FT-IR spectra of acid washed and untreated $\mathrm{SiO}_{2}$ rod is shown in Fig. S8. $\dagger$ Particularly, the CTAB and organic residues of untreated $\mathrm{SiO}_{2}$ rod were successfully removed by ethanolic acid washing supported from diminished intensity of carbon related peaks at $2980 \mathrm{~cm}^{-1}\left(\mathrm{C}-\mathrm{H}\right.$ stretching), $2920 \mathrm{~cm}^{-1}\left(\mathrm{CH}_{2}\right.$ scissoring mode), $2850 \mathrm{~cm}^{-1}$ (symmetric vibration of methylene chain), and $1450 \mathrm{~cm}^{-1}$ (symmetric vibration of methylene chain). As mentioned previously, ethanolic acid wash helped the uniform coating of $\mathrm{TiO}_{2}$ shell by removing the organic residues. Moreover, phase of materials was determined by X-ray diffraction (XRD) analysis (Fig. S7b $\dagger$ ). Only one broad peak was detected for all materials between $20^{\circ}$ and $30^{\circ}$, indicating the amorphous phase of sol-gel fabricated $\mathrm{SiO}_{2}, \mathrm{TiO}_{2}$, and its composite. $^{33,54}$ These results confirmed that the HST rod was successfully fabricated as a 1D, hollow, metal oxide composite material.

\subsection{Various intrinsic advantages of HST material}

A range of intrinsic properties were measured to confirm the advantageous characteristics of the HST rod. First, the dispersion stability (which is associated with pore volume and hollow spaces) of the $\mathrm{SiO}_{2}, \mathrm{ST} / \mathrm{CS}$, and HST rods was investigated (Fig. 5) ground materials were dispersed in silicone oil (viscosity $=100 \mathrm{cST}$ ) at a concentration of $3.0 \mathrm{wt} \%$ and the sedimentation ratio $(R)$ was determined. The dispersed materials gradually subsided and reached an equilibrium state after $90 \mathrm{~h}$. At first, the three materials showed similar sedimentation rates; however, after $10 \mathrm{~h}$ had passed, the core $\mathrm{SiO}_{2}$ rod and ST/ CS rod particles settled much more quickly than the HST rod material. After $90 \mathrm{~h}$, the HST material maintained an excellent sedimentation ratio of 0.9 , meaning that about $90 \%$ of the materials were still dispersed in the medium, while the ST/CS rod showed a sedimentation ratio of 0.77 and the core $\mathrm{SiO}_{2}$ rod showed 0.81 . This can be attributed to the enhanced porosity and pore volume of the HST material. Since HST materials are synthesized with large internal cavity, dispersion

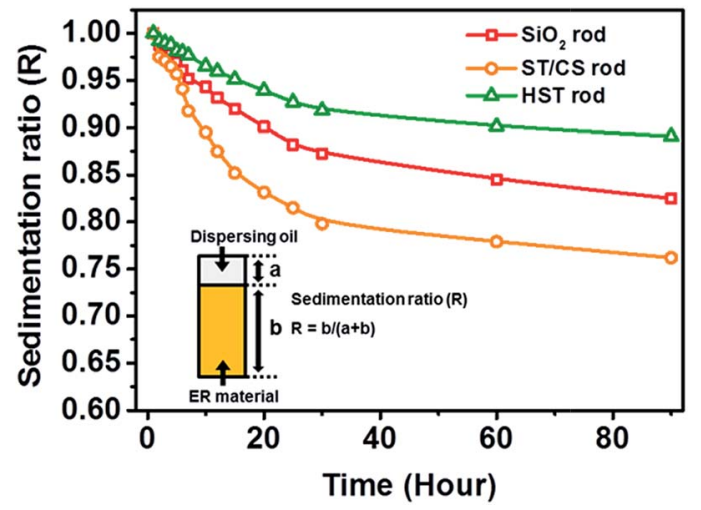

Fig. 5 Dispersion stability of $\mathrm{SiO}_{2}$ rod, ST/CS rod, and HST rod dispersed in silicone oil medium (inset: definition of sedimentation ratio). 
stability may have increased due to the enhanced pore volume. In contrast, the ST/CS rod showed the lowest value of $R$ due to the presence of both the $\mathrm{SiO}_{2}$ core and the $\mathrm{TiO}_{2}$ shell, which increased the particle density and mass. The actual particle densities of the three rod-like materials were determined using a pycnometer. The measured particle densities of the core $\mathrm{SiO}_{2}$, ST/CS, and HST rods were $2.77,3.01$, and $2.71 \mathrm{~g} \mathrm{~cm}^{-3}$, respectively. The sedimentation results were in good agreement with the measured particle densities. According to previous studies, a decrease in particle density can enhance the dispersion stability of a material and the particle mobility in a dispersing medium. ${ }^{50,55}$

The dielectric properties of the HST rod were investigated to confirm the advantages of the $\mathrm{TiO}_{2}$ (metal oxide) coating effect. The dielectric constants (or permittivity) $\left(\varepsilon^{\prime}\right)$ and loss factors $\left(\varepsilon^{\prime \prime}\right)$ of the three materials dispersed in silicone oil were measured as a function of electric field frequency $(f)$, as shown in Fig. 6. The dielectric constant of material is closely related to the polarizability of the material under an electric field; specifically, the achievable polarizability $(\Delta \varepsilon)$ and relaxation time $(\lambda)$ can be determined from the dielectric constant $\left(\varepsilon^{\prime}\right)$ and loss factor $\left(\varepsilon^{\prime \prime}\right)$ to investigate the polarization tendencies of a material. ${ }^{56}$ The achievable polarizability (magnitude of the polarization tendency) is derived from the difference between the fictitious $\left(\varepsilon_{0}\right)$ and static $\left(\varepsilon_{\infty}\right)$ dielectric constants. The former is measured in the low electric field frequency region $(f \rightarrow 0)$ and the latter is determined from the high frequency region $(f \rightarrow \infty)$. The measured achievable polarizabilities of the $\mathrm{SiO}_{2}, \mathrm{ST} / \mathrm{CS}$, and HST rods were 1.27, 1.98, and 3.05, respectively. The differences in achievable polarizability of the three rod-like materials were attributed to the combined effect of the $\mathrm{TiO}_{2}$ coating and increased surface area. In particular, the $\mathrm{TiO}_{2}$ coating increased the charge accumulation capacity and electrical conductivity and narrowed the band gap of the ST/CS and HST rods compared with those of the $\mathrm{SiO}_{2}$ rod, owing to the intrinsic and physical properties of $\mathrm{TiO}_{2}$. Moreover, the increased surface area of the HST rod also contributed to the increased charge accumulation capacity. The relaxation times $(\lambda)$ of the three

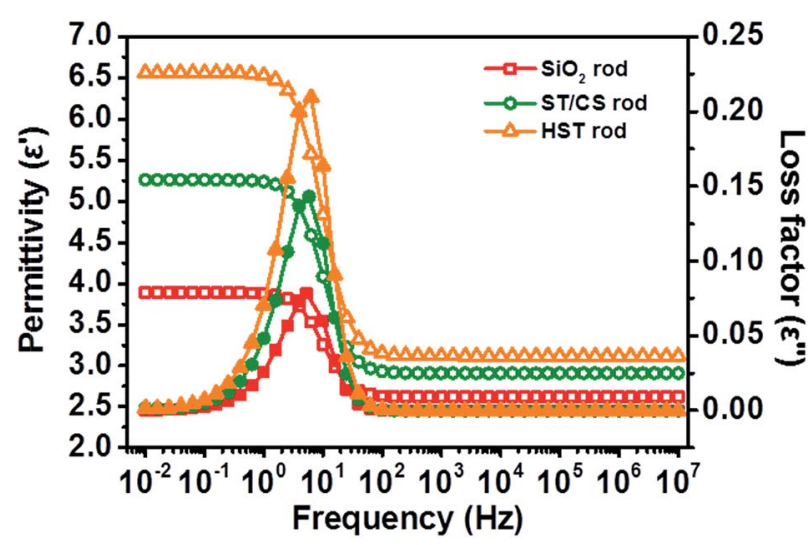

Fig. 6 Permittivity $\left(\varepsilon^{\prime}\right.$, open symbol) and loss factor ( $\varepsilon^{\prime \prime}$, closed symbol) of $\mathrm{SiO}_{2} \mathrm{rod}, \mathrm{ST} / \mathrm{CS}$ rod, and HST rod as a function of electric field frequency $(f)$. samples were measured to estimate the interfacial polarization efficiency of the materials using the following equation ${ }^{57}$

$$
\lambda=\frac{1}{2 \pi f_{\max }}
$$

where $f_{\max }$ is the maximum frequency peak of the dielectric loss factor. The $\lambda$ values determined for the $\mathrm{SiO}_{2}, \mathrm{ST} / \mathrm{CS}$, and HST rods were $0.031,0.027$, and $0.025 \mathrm{~s}$, respectively. Generally, a short relaxation time indicates a rapid polarization response of the material; ${ }^{58}$ therefore, it can be deduced that the HST rod materials possess more polarization capacity than the $\mathrm{SiO}_{2}$ and $\mathrm{ST} / \mathrm{CS}$ rods. Detailed dielectric properties of the $\mathrm{SiO}_{2}$ rod, ST/CS rod, and HST rod are listed in Table 1.

Considering these results, the HST rod was successfully fabricated by incorporation of the as-mentioned components to achieve various intrinsic and physical advantages, such as enhanced dielectric properties, dispersion stability, high porosity, and increased surface area. According to previous studies, each integrated component can provide useful properties for practical applications. For example, incorporation of the $\mathrm{TiO}_{2}$ composite provides photocatalytic activity, a rapid electron transportation layer for DSSC applications, and inhibition of bacterial growth..$^{21,23,59}$ In the case of hollow structures, possible applications are drug delivery, nanocages for encapsulation, and nanoreactors for material synthesis. ${ }^{29,60,61}$ The $1 \mathrm{D}$ metal oxide structure can be applied as a building block for nanodevices, provide mechanical strength by the overlapping of materials, and act as an electrical pathway. ${ }^{\mathbf{4 , 6 , 6 2}}$

\subsection{Electro-response of HST rod-based ER fluid}

In this study, we chose ER application to investigate the advantages of the as-synthesized HST rod. Generally, ER performance is affected by numerous factors, including applied electric field strength, temperature, electrode pattern, frequency of electric field, and properties of the material itself. ${ }^{\mathbf{4 0}-43}$ In recent decades, a huge number of materials with different compositions, sizes, and characteristics have been applied in ER studies to maximize performance. ${ }^{44-46}$ Among various characteristics, the most important factors for ER materials are dispersion stability (or anti-sedimentation property) and the dielectric property, which directly affects the ER activity. ${ }^{33}$ Thus, ER materials with a low particle mass and density are necessary to enhance dispersion stability. As

Table 1 Dielectric properties of $\mathrm{SiO}_{2}$ rod, ST/CS rod, and HST rod dispersed in silicone oil ${ }^{a}$

\begin{tabular}{lcclll}
\hline & $\varepsilon_{0}$ & $\varepsilon_{\infty}$ & $\Delta \varepsilon=\left(\varepsilon_{0}-\varepsilon_{\infty}\right)$ & $f_{\max }^{b}(\mathrm{~Hz})$ & $\Lambda^{c}(\mathrm{~s})$ \\
\hline $\mathrm{SiO}_{2} \operatorname{rod}$ & 2.62 & 3.89 & 1.27 & 5.30 & 0.03 \\
$\mathrm{ST} / \mathrm{CS}$ rod & 2.91 & 4.89 & 1.98 & 5.89 & 0.027 \\
HST rod & 3.12 & 6.17 & 3.05 & 6.32 & 0.025
\end{tabular}

${ }^{a}$ Dielectric parameters were acquired by impedance analyzer (Solatron 1260) coupled with dielectric interface (Solatron 1296). ${ }^{b}$ The $f_{\max }$ values of ER fluids were determined by nonlinear regression using OriginPro program. ${ }^{c}$ The relaxation time was determined using $\lambda=1 /\left(2 \pi f_{\max }\right)$ equation. 
mentioned previously, the HST rods were fabricated as a hollow material with a low particle density compared with non-hollow $\mathrm{SiO}_{2}$ and ST/CS rods, to attain enhanced dispersion stability. Moreover, the dielectric properties of the HST rod were greatly improved compared with those of the $\mathrm{SiO}_{2}$ and ST/CS rods owing to the synergistic effect of a high tailored surface area and $\mathrm{TiO}_{2}$ decoration. Previous studies also reported that geometric effects arising from 1D rod-like materials can improve ER performance. For instance, Lee et al. reported that rod-like GOcoated $\mathrm{SiO}_{2}$ materials exhibited superior ER efficiency compared with spherical GO-coated $\mathrm{SiO}_{2} \cdot{ }^{63}$ Moreover, Hong et al. provided a graphical explanation of the enhanced ER performance of $\mathrm{TiO}_{2}$ rod materials relative to that of $\mathrm{TiO}_{2}$ spheres. ${ }^{62}$ Specifically, the $1 \mathrm{D}$ rod-like material was able to exert excellent mechanical strength owing to the compact overlapping of materials. In this regard, our expectation was that the HST rod may be a good candidate for a ER material, combining the aforementioned advantages and fulfilling the requirements for a positive ER effect.

The shear stress curves of the $\mathrm{SiO}_{2}, \mathrm{ST} / \mathrm{CS}$, and HST rodbased ER fluids were examined as a function of shear rate $\left(\tau^{\prime}\right)$ under an applied electric field of $3.0 \mathrm{kV} \mathrm{mm}^{-1}$ (Fig. 7). By applying the electric field, all ER fluids manifested immediate shear stress curves due to the creation of fibril-like structures in response to the electrostatic force. In the low shear rate region, all three materials showed plateau curves representing Bingham-like behavior due to competition (and balancing) between the formation of fibril-like structures from the interfacial polarization and deformation caused by the hydrodynamic force. After passing the critical shear rate $\left(\tau_{\text {crit }}\right)$ near 100 $\mathrm{s}^{-1}$, Newtonian fluid behavior of proportional shear stress to shear rate was observed for all ER fluids. This was ascribed to the hydrodynamic force overcoming the electrostatic force, resulting in a sudden increase in shear stress. From this set of curves, it was clearly observed that the HST rod-based ER fluid demonstrated the best ER performance, followed by the ST/CS rod and then the $\mathrm{SiO}_{2}$ rod. In detail, the ST/CS rod exhibited higher ER activity than the $\mathrm{SiO}_{2}$ rod, even though the dispersion stability was slightly decreased by the $\mathrm{TiO}_{2}$ coating process. As

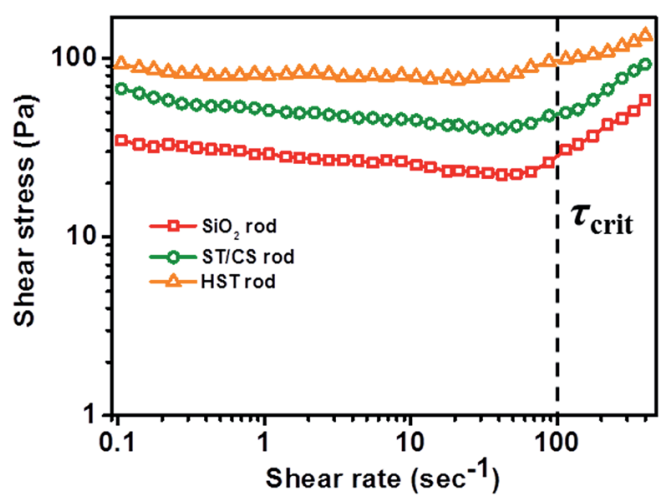

Fig. 7 Shear stress of $\mathrm{SiO}_{2}$ rod-, ST/CS rod-, and HST rod-based ER fluids as a function of shear rate $\left(\tau^{\prime}\right)$ under $3.0 \mathrm{kV} \mathrm{mm}^{-1}$ of electric field strength (3.0 wt\% in silicone oil). discussed earlier, this can be attributed to the increased dielectric property of materials incorporating the $\mathrm{TiO}_{2}$ shell. It was clear that synergistic contributions from the incorporation of $\mathrm{TiO}_{2}$ metal oxide and the increase in surface area effectively enhanced the interfacial polarizability of the ST/CS and HST rod materials. After the core $\mathrm{SiO}_{2}$ etching process, the ER activity of the HST rod was further improved compared with that of the ST/ CS rod due to enhanced dispersion stability and particle mobility originating from the large internal space created within the $\mathrm{TiO}_{2}$ shell. In general, ER materials with high dispersion stability can create more fibril-like structures to exert better ER performance by minimizing the sedimentation problem..$^{50}$ Hence, the optimal ER performance of the HST rodbased ER fluids can be understood to originate from low particle density, high porosity, and the presence of the hollow space, combined with the enhanced dielectric property of the material. Also, ER activities of HST rods with different $L / D$ were measured to investigate the influence of geometry on ER performance (Fig. S9†). Notably, ER performance of HST nanomaterials increased with increasing aspect ratio, but HST rod with $L / D$ of 5 showed decreased ER activity compared to rod with $L / D$ of 3 due to the aggregated states of material, as shown in Fig. S5.†

In addition, the rod-like geometric (1D orientation) effect of the HST rod on ER activity was assessed by direct comparison with spherical particles. For a precise comparison, spherical hollow $\mathrm{SiO}_{2} / \mathrm{TiO}_{2}$ (HST sphere) particles were synthesized using exactly the same method as that used for the HST rod, except $\mathrm{SiO}_{2}$ spheres were used as a core template material. Specifically, an HST sphere was fabricated with a diameter of $c a .75 \mathrm{~nm}$ (Fig. S5a $\dagger$ ) composed of the same elements ( $\mathrm{Si}, \mathrm{Ti}$, and $\mathrm{O}$ ) as the HST rod material (Table S4 $\uparrow$ ). Therefore, the HST spheres possessed similar characteristics and advantages to the HST rod materials, such as a hollow structure, high porosity, and a $\mathrm{TiO}_{2}$ shell; the only difference between the two materials was in their geometrical structures. The ER activities of the HST sphere and the HST rod were examined and compared, as shown in Fig. S9. $\dagger$ Noticeably, the HST sphere-based ER fluids exhibited maximum shear stress of $c a .16 \mathrm{~Pa}$, which was a 6-fold lower than that of the HST rod-based ER fluid, with maximum shear stress of $c a .93 \mathrm{~Pa}$. This result can be explained by various geometric effects originating from the 1D rod-like structure of the HST rod: flow resistance, mechanical stability, and polarization ability. First, the resistance of the HST rod to hydrodynamic force was greatly improved, owing to the rod-like structure of the material. The relationship between the flow resistance and the geometric structure of the material can be described in terms of dynamic drag $\left(F_{\mathrm{d}}\right)$ as follows: ${ }^{64}$

$$
F_{\mathrm{d}}=\frac{1}{2} \rho C_{\mathrm{d}} A v^{2}
$$

where $\rho$ is the density of the dispersing medium, $A$ is the crosssectional area of the dispersoid, $v$ is the relative velocity, and $C_{\mathrm{d}}$ represents the drag coefficient of the material. In general, $C_{\mathrm{d}}$ varies with the structural dimensions of dispersed materials. For example, $C_{\mathrm{d}}$ for spherical particles is known to be $\sim 0.47$, and this coefficient increases with elongation of the material 
dimensions. Therefore, 1D materials can exert higher flow resistance against mechanical shear to exhibit better ER performance in terms of drag force. Second, it is known that an array (or assembly) of elongated materials can provide better mechanical stability than spherical materials. ${ }^{32}$ In particular, dispersed materials form fibril-like structures along the direction of the applied electric field during ER activity, and the elongated 1D materials were able to form more rigid and stable fibril-like structures compared with spherical or short materials due to compact overlapping, which can prevent slippage between particles and provide better mechanical strength and stability within the fibril-like structures.

Finally, the polarization ability of a material is greatly influenced by the particle geometry. According to previous studies, 1D oriented (or rod-like) materials exhibited higher polarizability than spherical particles owing to the increased induced dipole moment. ${ }^{32,62,63,65}$ The magnitude of the dipole moment of spherical and ellipsoidal materials can be theoretically exploited by theories provided by Stratton and Dukhin. For a spherical particle, the dipole moment is determined by Stratton's equation: ${ }^{66,67}$

$$
\rho=4 \pi \varepsilon_{0} \varepsilon_{\mathrm{c}} a^{3} \beta E, \beta=\frac{\varepsilon_{\mathrm{p}}-\varepsilon_{\mathrm{c}}}{\varepsilon_{\mathrm{p}}+2 \varepsilon_{\mathrm{c}}}
$$

where $p$ is the magnitude of the dipole moment, $a$ represents the radius of a spherical particle, $E$ is the intensity of the electric field, $\beta$ is the relative polarizability of the particle, $\varepsilon_{0}$ is the vacuum permittivity $\left(8.854 \times 10^{-12} \mathrm{~F} \mathrm{~m}^{-1}\right), \varepsilon_{0}$ is the permittivity of the dispersing medium, and $\varepsilon_{\mathrm{p}}$ is the permittivity of the dispersed material. For a 1D ellipsoidal material, the dipole moment can be determined from the following equation given by Dukhin: ${ }^{68}$

$$
p=V \varepsilon_{0} \varepsilon_{\mathrm{c}} \frac{\varepsilon_{\mathrm{p}}-\varepsilon_{\mathrm{c}}}{\varepsilon_{\mathrm{c}}+\left(\varepsilon_{\mathrm{p}}-\varepsilon_{\mathrm{c}}\right) A_{\mathrm{z}}} E,
$$

where $p$ is the dipole moment, $V$ is the volume of the elongated material $\left(V=4 / 3 \pi a^{3} L / a\right)$, and $A_{\mathrm{z}}$ is the depolarization factor of material defined by the following equation:

$$
A_{\mathrm{z}}=\left(\xi^{2}-1\right)\left[\frac{\xi}{2} \ln \left(\frac{\xi+1}{\xi-1}\right)-1\right], \quad \xi=\frac{L}{\sqrt{L^{2}-a^{2}}},
$$

where $\xi$ is the geometrical factor, $L$ is the radius of the major axis of the material, and $a$ is the minor axis of the material. From these sets of equations, it can be deduced that the higher polarization ability of a 1D material originates from the increase in induced dipole moment. To support this theoretical explanation, the dielectric properties of HST spheres were also examined and compared with those of HST rods, and the practical polarization ability of the materials was verified (Fig. S10 $\dagger$ ). Furthermore, the achievable polarizability $(\Delta \varepsilon)$ and relaxation time $(\lambda)$ of the HST sphere were determined as 1.39 and $0.03 \mathrm{~s}$, respectively. From these results, the HST rod material with larger $\Delta \varepsilon(3.05)$ and shorter $\lambda(0.025 \mathrm{~s})$ has a better polarization ability and response than an HST sphere due to the increased dipole moment induced by the $1 \mathrm{D}$ structure. These experimental results confirm the advantage of rod-like materials over spherical ones in ER activity, originating from enhanced flow resistance, mechanical strength, and dielectric properties.

\subsection{Various stability and suitability test of HST-based ER fluid}

To investigate the stability and suitability of HST rod-based ER fluids, various characterizations were undertaken: ER activity as a function of electric field strength, electric field on-off test, and optical microscope analysis. The stability of the HST rod against an electric field was measured by varying the applied electric field strength at a fixed shear rate of $0.1 \mathrm{~s}^{-1}$, as shown in Fig. 8a. The applied electrical field strength was increased to $4.0 \mathrm{kV} \mathrm{mm}^{-1}$ in increments of $1.0 \mathrm{kV} \mathrm{mm}{ }^{-1}$. The three rod materials exhibited stable yield stresses without any fluctuation up to $4.0 \mathrm{kV} \mathrm{mm}^{-1}$, verifying their stability as ER fluids. In particular, the yield stress of materials increased in proportion to the square of the electric field strength in the low electric field region $\left(<1.0 \mathrm{kV} \mathrm{mm}^{-1}\right)$. In contrast, yield stresses increased with a 1.5 power of electric field strength in the high electric field region $\left(>1.0 \mathrm{kV} \mathrm{mm}^{-1}\right)$. From these results, ER fluids composed of HST rod and intermediate rod materials possess sufficient sustainability to withstand high electric field strengths without electrical shorting.

The continuousity, reversibility, and reproducibility of the materials were examined using an electric field on-off test (Fig. 8b). The applied electric field strength $\left(3.0 \mathrm{kV} \mathrm{mm}^{-1}\right)$ and shear rate $\left(0.1 \mathrm{~s}^{-1}\right)$ were completely fixed during this investigation; hence, the ER activity was solely controlled by the

a)

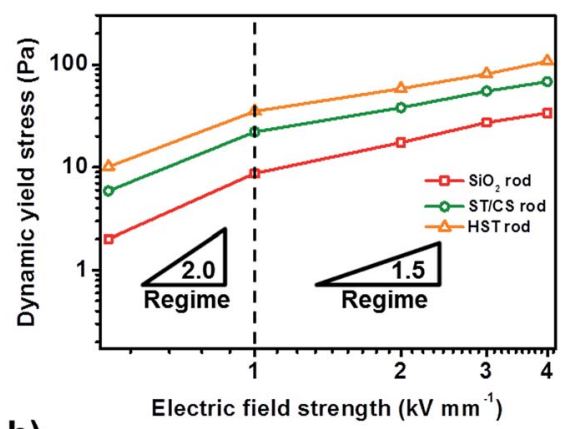

b)

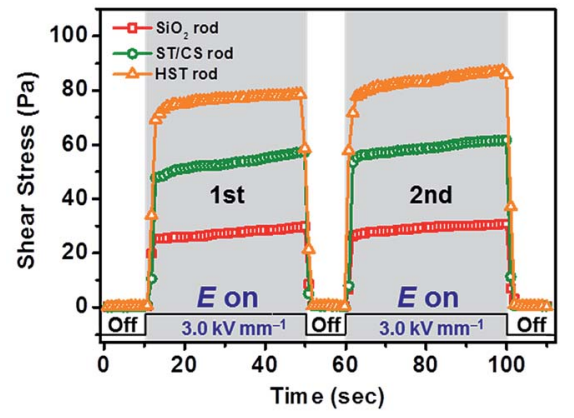

Fig. 8 (a) Yield stress of $\mathrm{SiO}_{2}$ rod-, ST/CS rod-, and HST rod-based ER fluids as a function of electric field strength $(E)$ with fixed shear rate of $0.1 \mathrm{~s}^{-1}$ (3.0 wt\% in silicone oil). (b) Electrical on-off test of $\mathrm{SiO}_{2}$ rod-, ST/CS rod-, and HST rod-based ER fluids with fixed shear rate of $0.1 \mathrm{~s}^{-1}$ $\left(E=3.0 \mathrm{kV} \mathrm{mm}^{-1}\right)$. 
presence of the electric field. Under the applied electric field, ER fluids exerted immediate shear stress curves. When the electric field was turned off, the shear stresses of all samples decreased almost immediately to their original state. Similar shear stress curves were observed in the second cycle, showing the reproducibility of ER fluids even after the application of large electric fields. Furthermore, the real-time structural changes of HST rod-based ER fluids were visualized using an optical microscope (OM) (Fig. S11 $\dagger$ ). First, well-dispersed ER fluids were placed between the electrodes to show randomly distributed particles. With the application of an electric field $\left(1.0 \mathrm{kV} \mathrm{mm}^{-1}\right)$, large numbers of rigid fibril-like structures were suddenly constructed within a few milliseconds to provide practical observation of ER activity. These results successfully demonstrated the stability, high reversibility, and reproducibility of HST rod materials for ER application.

\section{Conclusions}

A 1D oriented hollow $\mathrm{SiO}_{2} / \mathrm{TiO}_{2}$ (HST) rod was successfully synthesized via sequential steps of sol-gel use, shell coating, and the SMER method. The resulting HST rod particles manifested various advantages arising from the creation of an internal hollow space, incorporation of a $\mathrm{TiO}_{2}$ shell, and a rodlike structure. In particular, the hollow structure contributed to high pore volume, low density, and increased surface area. The $\mathrm{TiO}_{2}$ shell coating enhanced the dielectric property of the material through increased interfacial polarization and charge accumulation capacity. To obtain deeper insight into the synthesized material, the HST rod was adopted as an ER fluid, which requires various parameters for high performance. The HST rod exhibited 3.0- and 1.5-fold enhanced ER performance compared with the preliminary fabricated $\mathrm{SiO}_{2}$ and ST/CS rods due to the combined contributions of these properties. Moreover, HST rod-based ER fluids exhibited sixfold higher ER activity than similarly fabricated HST spheres due to enhanced mechanical strength, flow resistance, and dielectric property originating from the 1D structure. The ER application clearly demonstrated the numerous positive characteristics of HST rods, indicating that these versatile materials can be employed in various applications for future study.

\section{Acknowledgements}

This work was supported by Development Fund of Seoul National University funded by Dongjin Semichem Co., Korea (0458-20130066).

\section{Notes and references}

1 R. S. Devan, R. A. Patil, J.-H. Lin and Y.-R. Ma, Adv. Funct. Mater., 2012, 22, 3326.

2 T. Zhai, L. Li, Y. Ma, M. Liao, X. Wang, X. Fang, J. Yao, Y. Bando and D. Golberg, Chem. Soc. Rev., 2011, 40, 2986.

3 S. M. Liu, L. M. Gan, L. H. Liu, W. D. Zhang and H. C. Zeng, Chem. Mater., 2002, 14, 1391.
4 X. Duan, Y. Huang, Y. Cul, J. Wang and C. M. Liever, Nature, 2001, 409, 66.

5 Y. N. Xia, P. D. Yang, Y. G. Sun, Y. Y. Wu, B. Mayers, B. Gates, Y. D. Yin, F. Kim and H. Q. Yan, Adv. Mater., 2003, 15, 353.

6 C. N. R. Rao and A. Govindaraj, Adv. Mater., 2009, 21, 4208.

7 K. Lee, W. S. Seo and J. T. Park, J. Am. Chem. Soc., 2003, 125, 3408.

8 X. Fang, Y. Bando, U. K. Gautam, C. Ye and D. Golberg, J. Mater. Chem., 2008, 18, 509.

9 S. Hu and X. Wang, J. Am. Chem. Soc., 2008, 130, 8126.

10 K. R. Joshi and J. J. Schneider, Chem. Soc. Rev., 2012, 41, 5285.

11 Z. R. Dai, Z. W. Pan and Z. L. Wang, Adv. Funct. Mater., 2003, 13, 9.

12 Q. Zhang, H.-Y. Wang, X. Jia, B. Liu and Y. Yang, Nanoscale, 2013, 5, 7175.

13 T. Zhai, X. Fang, M. Liao, X. Xu, H. Zeng, B. Yoshino and D. Golberg, Sensors, 2009, 9, 6504.

14 A. Dev, J. P. Richters, J. Sartor, H. Kalt, J. Gutowski and T. Voss, Appl. Phys. Lett., 2011, 98, 131111.

15 S. V. N. T. Kuchibhatla, A. S. Karakoti, D. Bera and S. Seal, Prog. Mater. Sci., 2007, 52, 699.

16 M. Gutowski, J. E. Jaffe, C.-L. Liu, M. Stoker, R. I. Hedge, R. S. Rai and P. J. Tobin, Appl. Phys. Lett., 2002, 80, 1897.

17 X. Xia, J. Tu, Y. Zhang, X. Wang, C. Gu, X. Zhao and H. J. Fan, ACS Nano, 2012, 6, 5531.

18 B. C. Satishkumar, A. Govindaraj, M. Nath and C. N. R. Rao, J. Mater. Chem., 2000, 10, 2115.

19 A. I. Abdulagatov, Y. Yan, J. R. Cooper, Y. Zhang, Z. M. Gibbs, A. S. Cavanagh, R. G. Yang, Y. C. Lee and S. M. George, ACS Appl. Mater. Interfaces, 2011, 3, 4593.

20 J. Robertson, Eur. Phys. J.: Appl. Phys., 2004, 28, 265.

21 N. Wu, J. Wang, D. N. Tafen, H. Wang, J.-G. Zheng, J. P. Lewis, X. Liu, S. S. Leonard and A. Manivannan, J. Am. Chem. Soc., 2010, 132, 6679.

22 Z. Miao, D. Xu, J. Ouyang, G. Guo, X. Zhao and Y. Tang, Nano Lett., 2002, 2, 717.

23 D. Hwang, H. Lee, S.-Y. Jang, S. M. Jo, D. Kim, Y. Seo and D. Y. Kim, ACS Appl. Mater. Interfaces, 2011, 3, 2719.

24 K. M. Parida and B. Naik, J. Colloid Interface Sci., 2009, 333, 269.

25 W. Li and D. Zhao, Adv. Mater., 2013, 25, 142.

26 S. Son, S. H. Hwang, C. Kim, J. Yun and J. Jang, ACS Appl. Mater. Interfaces, 2013, 4, 4815.

27 J.-L. Hu, H.-S. Qian, J.-J. Li, Y. Hu, Z.-Q. Li and S.-H. Yu, Part. Part. Syst. Charact., 2013, 30, 306.

28 J. Lee, S. H. Hwang, J. Yun and J. Jang, ACS Appl. Mater. Interfaces, 2014, 6, 15420.

29 Y. Jang, S. Kim, W.-K. Oh, C. Kim, I. Lee and J. Jang, Chem. Commun., 2014, 50, 15345.

30 M. Choi, C. Kim, S. O. Jeon, K. S. Yook, J. Y. Lee and J. Jang, Chem. Commun., 2011, 47, 7092.

31 K. Lee, C.-M. Yoon, J. Noh and J. Jang, Chem. Commun., 2016, 52, 4231.

32 C.-M. Yoon, K. Lee, J. Noh, S. Lee and J. Jang, J. Mater. Chem. C, 2016, 4, 1713. 
33 C.-M. Yoon, S. Lee, O. J. Cheong and J. Jang, ACS Appl. Mater. Interfaces, 2015, 7, 18977.

34 S. H. Hwang, D. H. Shin, J. Yun, C. Kim, M. Choi and J. Jang, Chem.-Eur. J., 2014, 20, 4439.

35 J. Yun, S. H. Hwang and J. Jang, ACS Appl. Mater. Interfaces, 2015, 7, 2055.

36 J. B. Yin and X. P. Zhao, Chem. Mater., 2004, 16, 321.

37 M. Grzelczak, J. Vermant, E. M. Furst and L. M. Liz-Marzán, ACS Nano, 2010, 4, 3591.

38 S. Y. Oh, M. K. Oh and T. J. Kang, Colloids Surf., A, 2013, 436, 354.

39 J.-Y. Hong and J. Jang, Soft Matter, 2012, 8, 7348.

40 T. Hao, Adv. Mater., 2001, 13, 1847.

41 J. B. Yin and X. P. Zhao, Chem. Mater., 2002, 14, 4633.

42 J. Yin and X. Zhao, J. Phys. D: Appl. Phys., 2001, 34, 2063.

43 P. Gonon and J.-N. Foulc, J. Appl. Phys., 2000, 87, 3563.

44 C. McIntyre, H. Yang and P. F. Green, ACS Appl. Mater. Interfaces, 2013, 5, 8925.

45 Y. D. Liu, X. Quan, B. Hwang, Y. K. Kwon and H. J. Choi, Langmuir, 2014, 30, 1729.

46 S. D. Kim, W. L. Zhang and H. J. Choi, J. Mater. Chem. C, 2014, 2, 7541.

47 T. Hao, A. Kawai and F. Ikazaki, Langmuir, 1998, 14, 1256.

48 S. H. Kwon, S. H. Piao and H. J. Choi, Nanomaterials, 2015, 5, 2249.

49 L. D. Liu and H. Choi, Soft Matter, 2012, 8, 11961.

50 C.-M. Yoon, S. Lee, S. H. Hong and J. Jang, J. Colloid Interface Sci., 2015, 438, 14.

51 S. Kim, Y. Jang, W.-K. Oh, C. Kim and J. Jang, Adv. Healthcare Mater., 2014, 3, 1097.
52 X. Lu, M. Yu, G. Wang, T. Zhai, S. Xie, Y. Ling, Y. Tong and Y. Li, Adv. Mater., 2013, 25, 267.

53 A. N. Murashkevich, A. S. Lavitskaya, T. I. Barannikova and I. M. Zharskii, J. Appl. Spectrosc., 2008, 75, 730.

54 B. Ding, H. Kim, C. Kim, M. Khil and S. Park, Nanotechnology, 2003, 14, 532.

55 S. Lee, J. Noh, S. Hong, Y. K. Kim and J. Jang, Chem. Mater., 2016, 28, 2624.

56 J. P. Huang and K. W. Yu, Phys. Lett. A, 2004, 333, 347.

57 H. Block, J. P. Kelly, A. Qin and T. Watson, Langmuir, 1990, 6, 6.

58 B. X. Wang and X. P. Zhao, Adv. Funct. Mater., 2005, 15, 1815. 59 C. McCullagh, J. M. C. Robertson, D. W. Bahnemann and P. K. J. Robertson, Res. Chem. Intermed., 2007, 33, 359.

60 R. Kumar, A. N. Maitra, P. K. Patanjali and P. Sharma, Biomaterials, 2005, 26, 6743.

61 X. Lai, J. Li, B. A. Korgel, Z. Dong, Z. Li, F. Su, J. Du and D. Wang, Angew. Chem., 2011, 123, 2790.

62 J.-Y. Hong, M. Cho, C. Kim and J. Jang, J. Colloid Interface Sci., 2010, 347, 177.

63 S. Lee, C.-M. Yoon, J.-Y. Hong and J. Jang, J. Mater. Chem. C, 2014, 2, 6010.

64 Z.-G. Feng and E. E. Michaelides, J. Fluids Eng., 2001, 123, 841.

65 J. Noh, C.-M. Yoon and J. Jang, J. Colloid Interface Sci., 2016, 470, 237.

66 R. C. Kanu and M. T. Shaw, J. Rheol., 1998, 42, 657.

67 J. A. Stratton, Electromagnetic Theory, McGraws-Hill, New York and London, 1941.

68 S. S. Dukhin, Dielectric Properties of Disperse Systems, WileyInterscience, New York, 1971. 